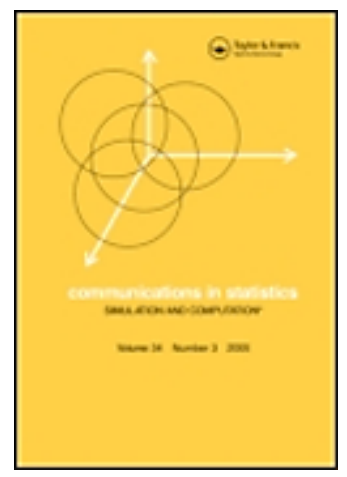

\title{
Discriminant Analysis for the von Mises-Fisher distribution
}

\begin{tabular}{|c|c|}
\hline Journal: & Communications in Statistics - Simulation and Computation \\
\hline Manuscript ID: & LSSP-2008-0319.R1 \\
\hline Manuscript Type: & Original Paper \\
\hline $\begin{array}{r}\text { Date Submitted by the } \\
\text { Author: }\end{array}$ & $11-J u l-2009$ \\
\hline Complete List of Authors: & $\begin{array}{l}\text { Figueiredo, Adelaide; Faculdade de Economia da Universidade do } \\
\text { Porto, Grupo de Matemática e Informática }\end{array}$ \\
\hline Keywords: & $\begin{array}{l}\text { Directional data, Discriminant Analysis, von Mises-Fisher } \\
\text { distribution }\end{array}$ \\
\hline Abstract: & $\begin{array}{l}\text { The von Mises-Fisher distribution is widely used for modeling } \\
\text { directional data. In this paper } \\
\text { we derive the discriminant rules based on } \\
\text { this distribution to assign objects into pre-existing classes. We } \\
\text { determine a distance between two von } \\
\text { Mises-Fisher populations and we calculate estimates of the } \\
\text { misclassification probabilities. We also analyse } \\
\text { the behavior of the distance between two von } \\
\text { Mises-Fisher populations and of the estimates of the } \\
\text { misclassification probabilities when we modify the parameters of } \\
\text { the populations or the samples size } \\
\text { or the dimension of the sphere. } \\
\text { Finally, we present an example with real spherical data available in } \\
\text { the literature. }\end{array}$ \\
\hline \multicolumn{2}{|c|}{$\begin{array}{l}\text { Note: The following files were submitted by the author for peer review, but cannot be converted } \\
\text { to PDF. You must view these files (e.g. movies) online. }\end{array}$} \\
\hline \multicolumn{2}{|c|}{ DiscriminantVMisesAdelaideFigueiredo.zip } \\
\hline
\end{tabular}




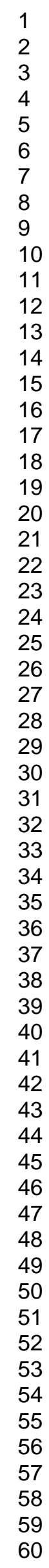

5) ScholaroNE" 


\title{
Discriminant Analysis for the von
}

\section{Mises-Fisher distribution}

\author{
Adelaide Figueiredo \\ Faculdade de Economia / LIAAD - INESC Porto L.A., Universidade do Porto \\ Rua Dr. Roberto Frias, 4200-464 Porto - PORTUGAL
}

\begin{abstract}
The von Mises-Fisher distribution is widely used for modeling directional data. In this paper we derive the discriminant rules based on this distribution to assign objects into pre-existing classes. We determine a distance between two von Mises-Fisher populations and we calculate estimates of the misclassification probabilities. We also analyse the behavior of the distance between two von Mises-Fisher populations and of the estimates of the misclassification probabilities when we modify the parameters of the populations or the samples size or the dimension of the sphere. Finally, we present an example with real spherical data available in the literature.
\end{abstract}

Keywords. Directional data; Discriminant Analysis; von Mises-Fisher distribution. AMS 2000 subject classification. $62 \mathrm{H} 11,62 \mathrm{H} 30$. 


\section{Introduction}

Directional statistics has been developed by many authors, for instance by Watson (1983), Mardia and Jupp (2000), Fisher, Lewis and Embleton (1987), Fisher (1993), Stephens (1982, 1992) and Jammalamadaka and SenGupta (2001). Discriminant Analysis for directional data was treated in the literature by Morris and Laycock (1974) and by El Khattabbi and Streit (1996) for circular data and spherical data. Figueiredo and Gomes (2006) considered discriminant analysis based on the Watson distribution defined on the hypersphere. The aim of this paper is to give discriminant rules based on the von Mises-Fisher distribution. We also determine a distance between two von Mises-Fisher populations with the same concentration parameter or distinct concentration parameters. As the misclassification probabilities cannot be obtained in closed form in the case of von Mises-Fisher populations, we determine estimates of these probabilities for several dimensions of the sphere, by generating samples from the von Mises-Fisher populations. For the particular case of the Fisher distribution, Morris and Laycock (1974) determined some expressions for the misclassification probabilities. We also analyse the behavior of the distance between the populations and of the misclassification probabilities estimates when we modify the parameters of the populations or the samples size or the dimension of the sphere.

In Sec. 2 we recall the von Mises-Fisher distribution; in Sec. 3 we consider discriminant analysis for the von Mises-Fisher distribution; in Sec. 4 we determine the distance between two von Mises-Fisher populations and the estimates of the misclassification probabilities for several values of the parameters of the populations, several samples sizes and several dimensions of the spheres; and finally in Sec. 5 we give an example with real spherical data. 


\section{2 von Mises-Fisher distribution}

The von Mises-Fisher distribution on the unit sphere in $\mathbb{R}^{p}, S_{p-1}=\left\{\mathbf{x} \in \mathbb{R}^{p}: \mathbf{x}^{\prime} \mathbf{x}=1\right\}$ is usually denoted by $M_{p}(\boldsymbol{\mu}, \kappa)$ and has probability density function given by

$$
f(\mathbf{x} ; \boldsymbol{\mu}, \kappa)=c_{p}(\kappa) \exp \left(\kappa \mathbf{x}^{\prime} \boldsymbol{\mu}\right) \quad \mathbf{x} \in S_{p-1}, \quad \boldsymbol{\mu} \in S_{p-1}, \kappa \geq 0,
$$

where the normalising constant is given by

$$
c_{p}(\kappa)=\frac{\kappa^{\frac{p}{2}-1}}{(2 \pi)^{\frac{p}{2}} I_{\frac{p}{2}-1}(\kappa)}
$$

with $I_{\nu}$ denoting the modified Bessel function of the first kind and order $\nu$ defined by

$$
I_{\nu}(\kappa)=\frac{1}{2 \pi} \int_{0}^{2 \pi} \cos \nu \theta e^{\kappa \cos \theta} \mathrm{d} \theta
$$

The parameter $\boldsymbol{\mu}$ is the mean direction and the parameter $\kappa$ specifies the concentration around $\boldsymbol{\mu}$. The parameter $\boldsymbol{\mu}$ is also the mode and $-\boldsymbol{\mu}$ is the antimode (provided that $\kappa>0$ ). The distribution is rotationally symmetric about $\boldsymbol{\mu}$ (Mardia and Jupp, 2000, p. 179).

The distribution $M_{p}(\boldsymbol{\mu}, \kappa)$ for the particular case of $p=3$ is called the Fisher distribution and for the particular case of $p=2$ is called the von Mises distribution.

If $\mathbf{x}$ comes from $M_{p}(\boldsymbol{\mu}, \kappa)$ and $U$ is a non-random orthogonal matrix, then $U \mathbf{x}$ comes from $M_{p}(U \boldsymbol{\mu}, \kappa)$

If $\mathbf{x}$ comes from $M_{p}(\boldsymbol{\mu}, \kappa)$ then for large $\kappa$ :

$$
2 \kappa\left(1-\mathbf{x}^{\prime} \boldsymbol{\mu}\right) \dot{\sim} \chi_{p-1}^{2}
$$

(See Mardia and Jupp, 2000, p. 172).

Let $\left(\mathbf{x}_{1}, \mathbf{x}_{2}, \ldots, \mathbf{x}_{n}\right)$ be a random sample of size $n$ from the von Mises-Fisher distribution $M_{p}(\boldsymbol{\mu}, \kappa)$. Let $\bar{R}$ be the mean resultant length of the sample defined by $\bar{R}=\|\overline{\mathbf{x}}\|=\left(\overline{\mathbf{x}}^{\prime} \overline{\mathbf{x}}\right)^{1 / 2}$, 
where $\overline{\mathbf{x}}$ is the sample vector mean of $\left(\mathbf{x}_{1}, \mathbf{x}_{2}, \ldots, \mathbf{x}_{n}\right)$ defined by $\overline{\mathbf{x}}=\sum_{i=1}^{n} \mathbf{x}_{i} / n$.

The maximum likelihood estimator of $\boldsymbol{\mu}$ is the sample mean direction, that is $\widehat{\boldsymbol{\mu}}=\overline{\mathbf{x}} /\|\overline{\mathbf{x}}\|$ and the maximum likelihood estimator of $\kappa$ is the solution of the equation $A_{p}(\kappa)=\|\overline{\mathbf{x}}\|$, where the function $A_{p}(\kappa)$ is defined by

$$
A_{p}(\kappa)=\frac{c_{p}^{\prime}(\kappa)}{c_{p}(\kappa)}=\frac{I_{\frac{p}{2}}(\kappa)}{I_{\frac{p}{2}-1}(\kappa)} .
$$

(See Mardia and Jupp, 2000, p. 198).

\section{Discriminant Analysis}

We suppose without loss of generality, two von Mises-Fisher populations, $P_{1}: M_{p}\left(\boldsymbol{\mu}_{1}, \kappa_{1}\right)$ and $P_{2}: M_{p}\left(\boldsymbol{\mu}_{2}, \kappa_{2}\right)$. Let $\left(\mathbf{x}_{i 1}, \ldots, \mathbf{x}_{i n_{i}}\right)$ be the sample from the population $i, i=1,2$. Next, we suppose that the parameters of the populations $\left(\boldsymbol{\mu}_{i}, \kappa_{i}\right), i=1,2$ are known. When these parameters are unknown, it is usual to replace them by their maximum likelihood estimates obtained from the respective samples.

The density function of the $i$ th population is given by

$$
f_{i}(\mathbf{x})=c_{p}\left(\kappa_{i}\right) \exp \left(\kappa_{i} \boldsymbol{\mu}_{i}^{\prime} \mathbf{x}\right), \quad \mathbf{x} \in S_{p-1}, \kappa_{i} \geq 0, \boldsymbol{\mu}_{i} \in S_{p-1}
$$

where the normalising constant $c_{p}\left(\kappa_{i}\right)$ is defined by $(2.2)$.

Our aim is to define a discriminant rule to assign a new object into one of the populations, that is, we want to divide the discriminant space into mutually exclusive and exhaustive regions $R_{1}$ and $R_{2}$, in order that any given object is classified into the population into whose region it falls.

Let $\pi_{i}(i=1,2)$ be the prior probability associated with the $i$ th population; $C(i \mid j)$ is the cost of classifying incorrectly an object from the $j$ th population into the $i$ th population $(i$, 
$j=1,2, i \neq j) ; P(i \mid j)$ is the probability of misclassifying an object from the $j$ th population into the $i$ th population $(i, j=1,2, i \neq j)$ defined by

$$
P(i \mid j)=P\left(\mathbf{x} \in R_{i} \mid \mathbf{x} \in P_{j}\right)=\int_{R_{i}} f_{j}(\mathbf{x}) \mathrm{d} \mathbf{x}
$$

and $C$ be the total cost of misclassification.

The Bayes rule that minimises the expected cost of misclassification $E(C)$ defined by $E(C)=C(2 \mid 1) P(2 \mid 1) \pi_{1}+C(1 \mid 2) P(1 \mid 2) \pi_{2}$ is the following:

Assign $\mathbf{x}$ to population 1 if

$$
C(2 \mid 1) f_{1}(\mathbf{x}) \pi_{1} \geq C(1 \mid 2) f_{2}(\mathbf{x}) \pi_{2}
$$

and to population 2, otherwise (see for instance, Johnson and Wichern, 1992, p. 557).

Substituting for $f_{1}(\mathbf{x})$ and $f_{2}(\mathbf{x})$ in (3.3), the Bayes rule is given by

Assign $\mathbf{x}$ to $M_{p}\left(\boldsymbol{\mu}_{1}, \kappa_{1}\right)$ if

$$
\left(\kappa_{1} \boldsymbol{\mu}_{1}-\kappa_{2} \boldsymbol{\mu}_{2}\right)^{\prime} \mathbf{x}+\ln \frac{c_{p}\left(\kappa_{1}\right)}{c_{p}\left(\kappa_{2}\right)} \geq \ln \frac{C(1 \mid 2) \pi_{2}}{C(2 \mid 1) \pi_{1}}
$$

and to $M_{p}\left(\boldsymbol{\mu}_{2}, \kappa_{2}\right)$ otherwise.

Denoting the discriminant function by $W_{12}$, i.e.

$$
W_{12}=\left(\kappa_{1} \boldsymbol{\mu}_{1}-\kappa_{2} \boldsymbol{\mu}_{2}\right)^{\prime} \mathbf{x}+\ln \frac{c_{p}\left(\kappa_{1}\right) C(2 \mid 1) \pi_{1}}{c_{p}\left(\kappa_{2}\right) C(1 \mid 2) \pi_{2}}
$$

the discriminant regions $R_{1}$ and $R_{2}$ are given as

$$
\begin{aligned}
& R_{1}=\left\{\mathbf{x} \in S_{p-1}: W_{12} \geq 0\right\} \\
& R_{2}=\left\{\mathbf{x} \in S_{p-1}: W_{12}<0\right\} .
\end{aligned}
$$

A measure of the distance between two populations, referred in Morris and Laycock (1974) and used by El Khattabi and Streit (1996), which is equivalent to Mahalonobis's $D^{2}$ 
in the multivariate normal case, is defined by

$$
D=E\left(W_{12} \mid \mathbf{x} \in P_{1}\right)-E\left(W_{12} \mid \mathbf{x} \in P_{2}\right) .
$$

If $\mathbf{x}$ comes from the von Mises-Fisher distribution $M_{p}(\boldsymbol{\mu}, \kappa)$, then

$$
E(\mathbf{x})=\rho \boldsymbol{\mu},
$$

where $\rho=A_{p}(k)$ with the function $A_{p}(\kappa)$ defined by (2.5) (Mardia and Jupp, 2000, p. 169). Note that for $p=3$, the function $A_{p}(k)$ may be calculated as $A_{p}(k)=\operatorname{coth} \kappa-\frac{1}{\kappa}$ (Mardia and Jupp, 2000, p. 169).

Then,

$$
\begin{aligned}
& E\left(W_{12} \mid \mathbf{x} \in P_{1}\right)=\ln \frac{c_{p}\left(\kappa_{1}\right) C(2 \mid 1) \pi_{1}}{c_{p}\left(\kappa_{2}\right) C(1 \mid 2) \pi_{2}}+A_{p}\left(\kappa_{1}\right)\left(\kappa_{1} \boldsymbol{\mu}_{1}-\kappa_{2} \boldsymbol{\mu}_{2}\right)^{\prime} \boldsymbol{\mu}_{1} \\
& E\left(W_{12} \mid \mathbf{x} \in P_{2}\right)=\ln \frac{c_{p}\left(\kappa_{1}\right) C(2 \mid 1) \pi_{1}}{c_{p}\left(\kappa_{2}\right) C(1 \mid 2) \pi_{2}}+A_{p}\left(\kappa_{2}\right)\left(\kappa_{1} \boldsymbol{\mu}_{1}-\kappa_{2} \boldsymbol{\mu}_{2}\right)^{\prime} \boldsymbol{\mu}_{2}
\end{aligned}
$$

and consequently, the distance $D$ is given by

$$
D=\left(\kappa_{1} \boldsymbol{\mu}_{1}-\kappa_{2} \boldsymbol{\mu}_{2}\right)^{\prime}\left(A_{p}\left(\kappa_{1}\right) \boldsymbol{\mu}_{1}-A_{p}\left(\kappa_{2}\right) \boldsymbol{\mu}_{2}\right) \text {. }
$$

If the two populations have the same concentration parameter, i.e., $\kappa_{1}=\kappa_{2}=\kappa$, the distance $D$ simplifies to

$$
D=\kappa A_{p}(\kappa)\left(\boldsymbol{\mu}_{1}-\boldsymbol{\mu}_{2}\right)^{\prime}\left(\boldsymbol{\mu}_{1}-\boldsymbol{\mu}_{2}\right) .
$$

The probabilities of misclassification are defined by

$$
\begin{gathered}
P(1 \mid 2)=P\left(W_{12} \geq 0 \mid \mathbf{x} \in P_{2}\right) \\
P(2 \mid 1)=P\left(W_{12}<0 \mid \mathbf{x} \in P_{1}\right) .
\end{gathered}
$$

As we can not obtain any closed expressions for these misclassification probabilities in the case of the von Mises-Fisher distribution, we estimate them in some cases in the next 
section. These probabilities can also be represented, as an integral, which can be calculated numerically. Morris and Laycock (1974) expressed these misclassification probabilities in terms of an integral in the cases of $p=2$ and $p=3$. In the next section we determine the misclassification probabilities for $p=2$. For the calculation of the integrals we have used the algorithm proposed in Hill (1977) or alternatively, we could have used the tables of the distribution function for the von Mises distribution given for instance, in Mardia and Jupp (2000), Appendix 2.

For $k$ von Mises-Fisher populations, the discriminant functions are given by

$$
W_{j i}=\left(\kappa_{j} \boldsymbol{\mu}_{j}-\kappa_{i} \boldsymbol{\mu}_{i}\right)^{\prime} \mathbf{x}+\ln \frac{c_{p}\left(\kappa_{j}\right) C(i \mid j) \pi_{j}}{c_{p}\left(\kappa_{i}\right) C(j \mid i) \pi_{i}}, \quad i, j=1, \ldots, k, i \neq j
$$

and the discriminant regions are defined by

$$
R_{j}=\left\{\mathbf{x} \in S_{p-1}: W_{j i} \geq 0, i=1, \ldots, k, i \neq j\right\}
$$

It is enough to use $k-1$ functions for defining the discriminant regions, provided that $k-1<p$; we may use for instance, the discriminant functions $W_{1 j}, j=2, \ldots, k$.

We have not presented the previous results for the particular cases of $p=2$ and $p=3$ because they were already considered in Morris and Laycock (1974).

\section{Estimates of the misclassification probabilities and distance between the two populations}

We have investigated the above procedures by a large Monte Carlo study. We have considered two von Mises-Fisher populations $P_{1}: M_{p}\left(\boldsymbol{\mu}_{1}, \kappa_{1}\right)$ and $P_{2}: M_{p}\left(\boldsymbol{\mu}_{2}, \kappa_{2}\right)$, with known parameters. We have supposed the two populations with equal prior probabilities: $\pi_{1}=\pi_{2}=0.5$ and equal costs of misclassification: $C(1 \mid 2)=C(2 \mid 1)$. We have considered the 
following dimensions of the sphere: $p=2,3,4,10$, and for each $p$, we have taken the mean directions: $\boldsymbol{\mu}_{1}=(0, \ldots, 0,1)^{\prime}$ and $\boldsymbol{\mu}_{2}=\left(0,0, \ldots,\left(1-\cos ^{2} \theta\right)^{1 / 2}, \cos \theta\right)^{\prime}$, supposing that the angle $\theta$ between these directions is equal to $0^{\circ}\left(30^{\circ}\right) 180^{\circ}$. The results only depend on $\boldsymbol{\mu}_{1}$ and $\boldsymbol{\mu}_{2}$ through their angular separation $\theta=\operatorname{acos}\left(\boldsymbol{\mu}_{1}^{\prime} \cdot \boldsymbol{\mu}_{2}\right)$. We have supposed the following two cases:

- Populations with equal concentration parameters: $\kappa_{1}=\kappa_{2}=\kappa$, with $\kappa=(3), 5,10,15(20)$.

- Populations with different concentration parameters: $\kappa_{1} \neq \kappa_{2}, \kappa_{1}=5, \kappa_{2}=10,15,20(25)$. (We have considered only $\kappa_{1}<\kappa_{2}$, because if $\kappa_{1}>\kappa_{2}$, the distance $D$ and the estimates of the misclassification probabilities are identical).

For both cases, we have estimated the misclassification probabilities $P(2 \mid 1)$ and $P(1 \mid 2)$ by the misclassification proportions in 10000 observations generated from $P_{1}$ and $P_{2}$ populations, respectively, and then we have calculated the average of these proportions to obtain an estimate of the total misclassification probability. When the two populations have the same concentration parameter $\kappa_{1}=\kappa_{2}$, then the misclassification probabilities $P(2 \mid 1)$ and $P(1 \mid 2)$ are equal (or approximately equal, if these are estimated), but when $\kappa_{1} \neq \kappa_{2}$, the misclassification probabilities are not equal because in this case, the probability of assigning an observation to the population with greater concentration parameter is the larger (see the expression (4.2)).

For generating observations from the von Mises-Fisher distribution, we have used the method given in Wood (1994). 


\subsection{Populations with equal concentration parameters}

In this case the discriminant function defined by (3.5) reduces to

$$
W_{12}=\kappa\left(\boldsymbol{\mu}_{1}-\boldsymbol{\mu}_{2}\right)^{\prime} \mathbf{x}
$$

The values obtained for the distance $D$ defined by (3.8) and for the estimates of the misclassification probabilities for some values of the angle $\theta\left(^{\circ}\right)=30,60,(90) 120,(180)$ and $\kappa=(3), 5,10,15,(20)$ are indicated for $p=2,3,4$ in Tables 1-3. See also figure 1.

As expected, for each $p$ and fixed $\theta$, when $\kappa$ increases, the estimated misclassification probabilities decrease, tending very quickly to 0 and the distance $D$ between the populations increases.

For each $p$ and fixed $\kappa$ when the angle $\theta$ increases, the estimates of the misclassification probabilities decrease and the distance $D$ between the populations increases.

For fixed $\kappa$ and $\theta$, as the dimension of the sphere $p$ increases, the estimated misclassification probabilities increase and the distance $D$ between the populations decreases.

We have also observed that in the case of $p=2$, for each $\theta, \kappa$, the estimated misclassification probabilities are close from the theorical misclassification probabilities.

\subsection{Populations with different concentration parameters}

In this case the discriminant function defined by (3.5) reduces to

$$
W_{12}=\left(\kappa_{1} \boldsymbol{\mu}_{1}-\kappa_{2} \boldsymbol{\mu}_{2}\right)^{\prime} \mathbf{x}+\ln \frac{c_{p}\left(\kappa_{1}\right)}{c_{p}\left(\kappa_{2}\right)} .
$$

The values obtained for the distance $D$ defined by (3.7) and for the estimates of the misclassification probabilities for for some values of the angle $\theta\left(^{\circ}\right)=30,60,(90) 120,(180)$ and $\kappa_{1}=5, \kappa_{2}=10,15,20(25)$, are indicated for $p=2,3,4$ in Tables 4-6. See also figure 2 . 
Table 1: Misclassification probabilities and distance $D$ between the two populations for $p=2$

\begin{tabular}{|c|c|c|c|c|}
\hline$\theta\left(^{o}\right)$ & $\kappa$ & $\widehat{P(1 \mid 2)}=\widehat{P(2 \mid 1)}$ & $P(1 \mid 2)=P(2 \mid 1)$ & $D$ \\
\hline & 3 & 0.384 & 0.334 & 0.036 \\
\hline \multirow{4}{*}{30} & 5 & 0.278 & 0.285 & 0.317 \\
\hline & 10 & 0.156 & 0.208 & 1.309 \\
\hline & 15 & 0.099 & 0.158 & 2.374 \\
\hline & 3 & 0.187 & 0.197 & 1.299 \\
\hline \multirow[t]{4}{*}{60} & 5 & 0.094 & 0.131 & 3.017 \\
\hline & 10 & 0.026 & 0.053 & 7.431 \\
\hline & 15 & 0.009 & 0.023 & 11.947 \\
\hline & 3 & 0.098 & 0.104 & 3.556 \\
\hline \multirow[t]{4}{*}{90} & 5 & 0.032 & 0.049 & 7.192 \\
\hline & 10 & 0.003 & 0.009 & 16.480 \\
\hline & 15 & 0 & 0.002 & 25.914 \\
\hline & 3 & 0.056 & 0.049 & 6.392 \\
\hline \multirow[t]{3}{*}{120} & 5 & 0.012 & 0.015 & 12.252 \\
\hline & 10 & 0 & 0.001 & 26.722 \\
\hline & 15 & 0 & 0 & 41.263 \\
\hline
\end{tabular}

For each dimension of the sphere $p$, for fixed $\kappa_{1}$ and $\theta$, when $\kappa_{2}$ increases, the misclassification probabilities estimates decrease and the distance $D$ between the populations 
Table 2: Estimates of the misclassification probabilities and distance $D$ between the two populations for $p=3$

\begin{tabular}{|c|c|c|c|c|c|}
\hline$\theta\left(^{o}\right)$ & $\kappa$ & $\widehat{P(1 \mid 2)}$ & $\widehat{P(2 \mid 1)}$ & Average & $D$ \\
\hline \multirow{4}{*}{30} & 5 & 0.298 & 0.298 & 0.298 & 1.072 \\
\hline & 10 & 0.216 & 0.215 & 0.216 & 2.411 \\
\hline & 15 & 0.162 & 0.161 & 0.162 & 3.751 \\
\hline & 20 & 0.126 & 0.126 & 0.126 & 5.091 \\
\hline \multirow{4}{*}{60} & 5 & 0.149 & 0.145 & 0.147 & 4.000 \\
\hline & 10 & 0.060 & 0.059 & 0.060 & 9.000 \\
\hline & 15 & 0.026 & 0.026 & 0.026 & 13.999 \\
\hline & 20 & 0.012 & 0.012 & 0.012 & 18.999 \\
\hline \multirow{4}{*}{120} & 5 & 0.024 & 0.023 & 0.024 & 12.001 \\
\hline & 10 & 0.001 & 0.001 & 0.001 & 27.000 \\
\hline & 15 & 0 & 0 & 0 & 41.999 \\
\hline & 20 & 0 & 0 & 0 & 56.999 \\
\hline \multirow{4}{*}{180} & 5 & 0.007 & 0.007 & 0.007 & 16.002 \\
\hline & 10 & 0 & 0 & 0 & 36.000 \\
\hline & 15 & 0 & 0 & 0 & 56.000 \\
\hline & 20 & 0 & 0 & 0 & 76.000 \\
\hline
\end{tabular}

increases.

For each $p$, for fixed $\kappa_{1}$ and $\kappa_{2}$, as the angle $\theta$ increases, the estimated misclassification probabilities decrease and the distance $D$ between the populations increases. 
Table 3: Estimates of the misclassification probabilities and distance $D$ between the two populations for $p=4$

\begin{tabular}{cccccc}
$\theta\left(^{o}\right)$ & $\kappa$ & $\widehat{P(1 \mid 2)}$ & $\widehat{P(2 \mid 1)}$ & Average & $D$ \\
\hline 5 & 0.308 & 0.308 & 0.308 & 0.963
\end{tabular}

$\begin{array}{llllll}30 & 10 & 0.219 & 0.223 & 0.221 & 2.288\end{array}$

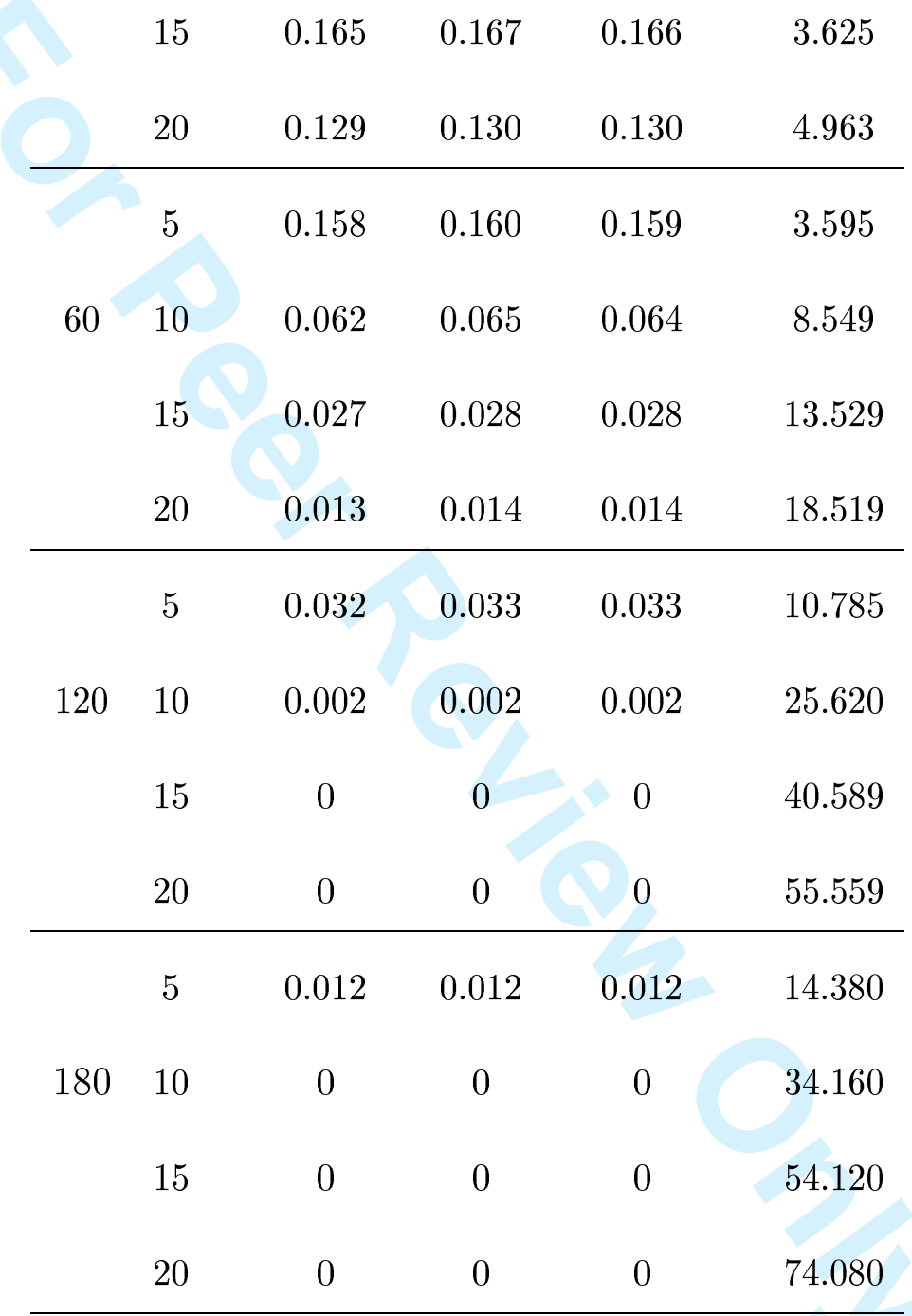

For fixed $\kappa_{1}, \kappa_{2}, \theta$, when $p$ increases, the estimates of the misclassification probabilities increase and the distance $D$ between the populations decreases.

We have also observed that in the case of $p=2$, for each $\theta, \kappa_{1}, \kappa_{2}$ and unless $\theta=30^{\circ}$, 
Angle 90은

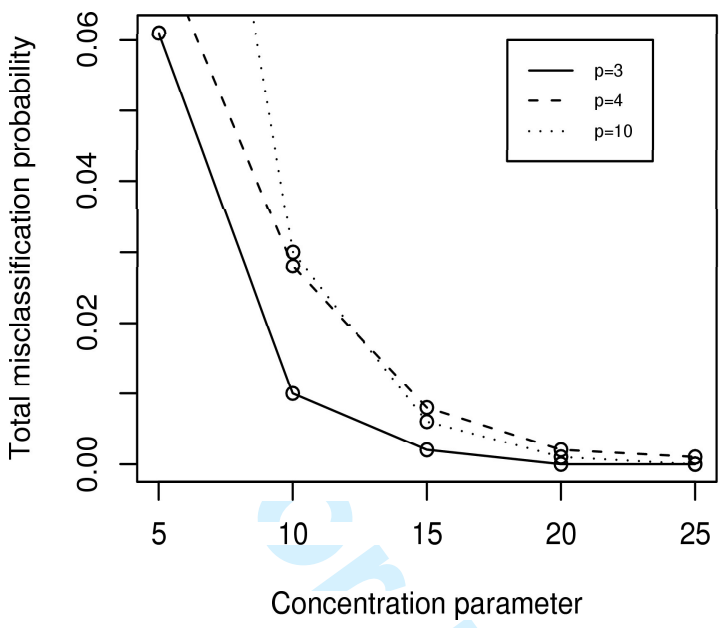

Concentration parameter 10

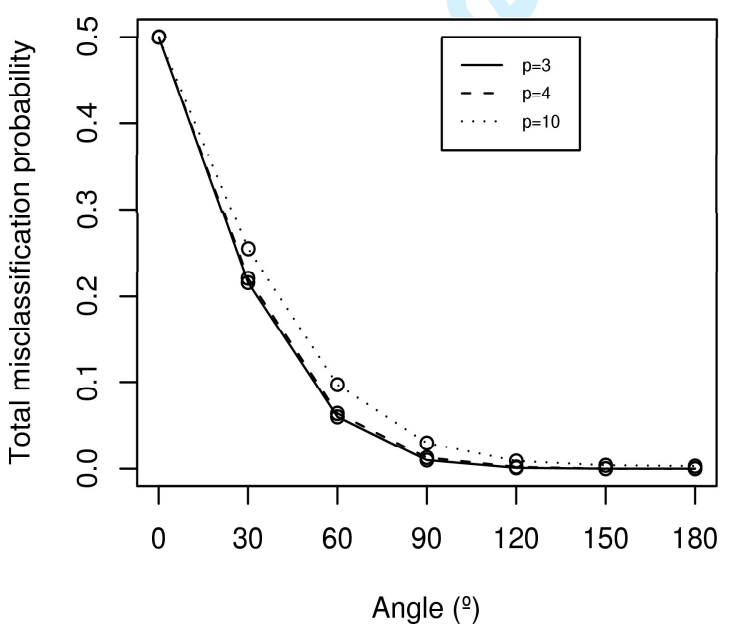

Angle $90^{\circ}$

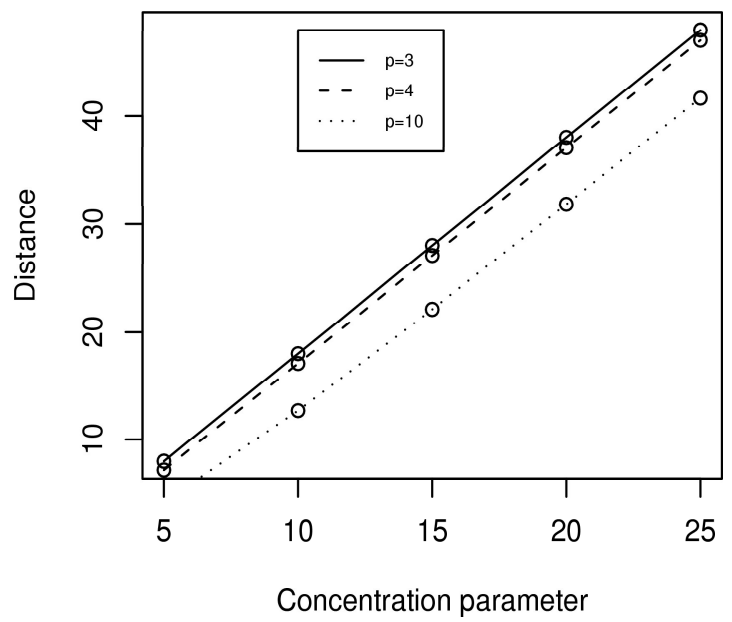

Concentration parameter 10

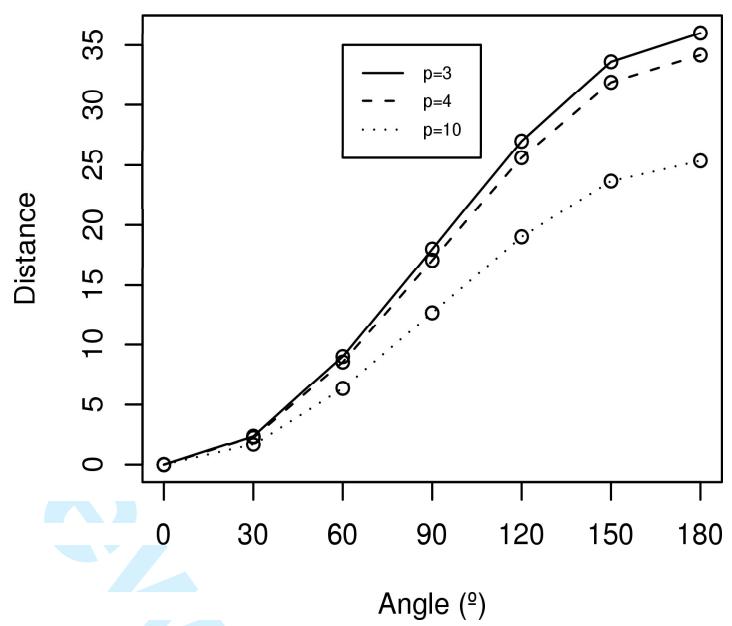

Figure 1: Estimate of the total misclassification probability and distance $D$ between the two populations

the estimated misclassification probabilities are close from the theorical misclassification probabilities. 
Table 4: Misclassification probabilities and distance $D$ between the two populations for $p=2$

\begin{tabular}{|c|c|c|c|c|c|c|c|c|c|}
\hline$\theta\left(^{o}\right)$ & $\kappa_{1}$ & $\kappa_{2}$ & $\widehat{P(1 \mid 2)}$ & $\widehat{P(2 \mid 1)}$ & Average & $P(1 \mid 2)$ & $P(2 \mid 1)$ & Average & $D$ \\
\hline \multirow{3}{*}{30} & \multirow{3}{*}{5} & 10 & 0.114 & 0.144 & 0.129 & 0.253 & 0.321 & 0.287 & 1.141 \\
\hline & & 15 & 0.069 & 0.084 & 0.077 & 0.200 & 0.312 & 0.256 & 1.794 \\
\hline & & 20 & 0.049 & 0.050 & 0.050 & 0.153 & 0.298 & 0.225 & 2.414 \\
\hline \multirow{3}{*}{60} & \multirow{3}{*}{5} & 10 & 0.034 & 0.012 & 0.023 & 0.041 & 0.113 & 0.077 & 5.710 \\
\hline & & 15 & 0.019 & 0.002 & 0.011 & 0.011 & 0.098 & 0.055 & 8.144 \\
\hline & & 20 & 0.012 & 0 & 0.007 & 0.003 & 0.088 & 0.045 & 10.538 \\
\hline \multirow{3}{*}{90} & \multirow{3}{*}{5} & 10 & 0.012 & 0.001 & 0.007 & 0.004 & 0.031 & 0.017 & 12.360 \\
\hline & & 15 & 0.007 & 0 & 0.004 & 0 & 0.023 & 0.011 & 17.276 \\
\hline & & 20 & 0.005 & 0 & 0.003 & 0 & 0.018 & 0.009 & 22.146 \\
\hline \multirow{3}{*}{120} & \multirow{3}{*}{5} & 10 & 0.002 & 0 & 0.001 & 0 & 0.007 & 0.004 & 20.097 \\
\hline & & 15 & 0 & 0 & 0 & 0 & 0.004 & 0.002 & 27.579 \\
\hline & & 20 & 0 & 0 & 0 & 0 & 0.003 & 0.001 & 34.989 \\
\hline
\end{tabular}

\section{Example}

We use the spherical data given in Wood (1982) (Table 2 in Schmidt, 1976) consisting of a set of 33 estimates of a previous magnetic pole position of the earth obtained using palaeomagnetic techniques. Each estimate is associated with a different site, the 33 sites being spread over a large area of Tasmania. Schmidt says that "the data appear to fall into two main groups which are derived from two distinct geographical regions" . 
Table 5: Estimates of the misclassification probabilities and distance $D$ between the two populations for $p=3$

\begin{tabular}{|c|c|c|c|c|c|c|}
\hline$\theta\left(^{o}\right)$ & $\kappa_{1}$ & $\kappa_{2}$ & $\widehat{P(1 \mid 2)}$ & $\widehat{P(2 \mid 1)}$ & Average & $D$ \\
\hline \multirow{4}{*}{30} & \multirow{4}{*}{5} & 10 & 0.188 & 0.305 & 0.247 & 2.325 \\
\hline & & 15 & 0.136 & 0.270 & 0.203 & 3.566 \\
\hline & & 20 & 0.107 & 0.240 & 0.174 & 5.260 \\
\hline & & 25 & 0.088 & 0.215 & 0.152 & 6.520 \\
\hline \multirow{4}{*}{60} & \multirow{4}{*}{5} & 10 & 0.084 & 0.117 & 0.101 & 6.750 \\
\hline & & 15 & 0.053 & 0.094 & 0.074 & 9.658 \\
\hline & & 20 & 0.036 & 0.079 & 0.058 & 12.630 \\
\hline & & 25 & 0.026 & 0.068 & 0.047 & 15.590 \\
\hline \multirow{4}{*}{120} & \multirow{4}{*}{5} & 10 & 0.009 & 0.009 & 0.009 & 19.250 \\
\hline & & 15 & 0.004 & 0.005 & 0.005 & 26.327 \\
\hline & & 20 & 0.002 & 0.003 & 0.003 & 33.380 \\
\hline & & 25 & 0.001 & 0.002 & 0.002 & 40.390 \\
\hline \multirow{4}{*}{180} & \multirow{4}{*}{5} & 10 & 0.001 & 0.002 & 0.002 & 25.500 \\
\hline & & 15 & 0 & 0.001 & 0.001 & 34.660 \\
\hline & & 20 & 0 & 0 & 0 & 43.750 \\
\hline & & 25 & 0 & 0 & 0 & 52.800 \\
\hline
\end{tabular}

In discriminant analysis we need to know a priori the groups of observations. The EM (Estimation-Maximisation) algorithm proposed by Dempster, Laird and Rubin (1977) can be used in many contexts and in particular, to estimate the parameters of a mixture of 15 
Table 6: Estimates of the misclassification probabilities and distance $D$ between the two populations for $p=4$

\begin{tabular}{ccccccc}
\hline$\theta\left(^{o}\right)$ & $\kappa_{1}$ & $\kappa_{2}$ & $\widehat{P(1 \mid 2)}$ & $\widehat{P(2 \mid 1)}$ & Average & $D$ \\
\hline & 10 & 0.204 & 0.287 & 0.246 & 1.066
\end{tabular}

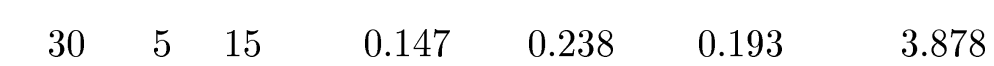

\begin{tabular}{|c|c|c|c|c|c|c|}
\hline & & 20 & 0.112 & 0.199 & 0.156 & 6.483 \\
\hline & & 25 & 0.088 & 0.171 & 0.130 & 7.486 \\
\hline \multirow{4}{*}{60} & \multirow{4}{*}{5} & 10 & 0.100 & 0.120 & 0.110 & 6.408 \\
\hline & & 15 & 0.066 & 0.092 & 0.079 & 9.475 \\
\hline & & 20 & 0.046 & 0.072 & 0.059 & 15.171 \\
\hline & & 25 & 0.034 & 0.059 & 0.047 & 15.777 \\
\hline \multirow{4}{*}{120} & \multirow{4}{*}{5} & 10 & 0.014 & 0.013 & 0.014 & 17.868 \\
\hline & & 15 & 0.007 & 0.007 & 0.007 & 24.770 \\
\hline & & 20 & 0.004 & 0.004 & 0.004 & 31.621 \\
\hline & & 25 & 0.003 & 0.003 & 0.003 & 38.470 \\
\hline \multirow{4}{*}{180} & \multirow{4}{*}{5} & 10 & 0.002 & 0.003 & 0.003 & 23.595 \\
\hline & & 15 & 0.001 & 0.001 & 0.001 & 32.420 \\
\hline & & 20 & 0 & 0.001 & 0.001 & 41.125 \\
\hline & & 25 & 0 & 0 & 0 & 49.800 \\
\hline
\end{tabular}

distributions, more precisely to solve the likelihood equations. This algorithm also enables us to obtain a partition of data into groups, which are required in discriminant analysis. So, we have used this algorithm in this example to obtain a partition of the data into two groups 
Dimension of the sphere 3

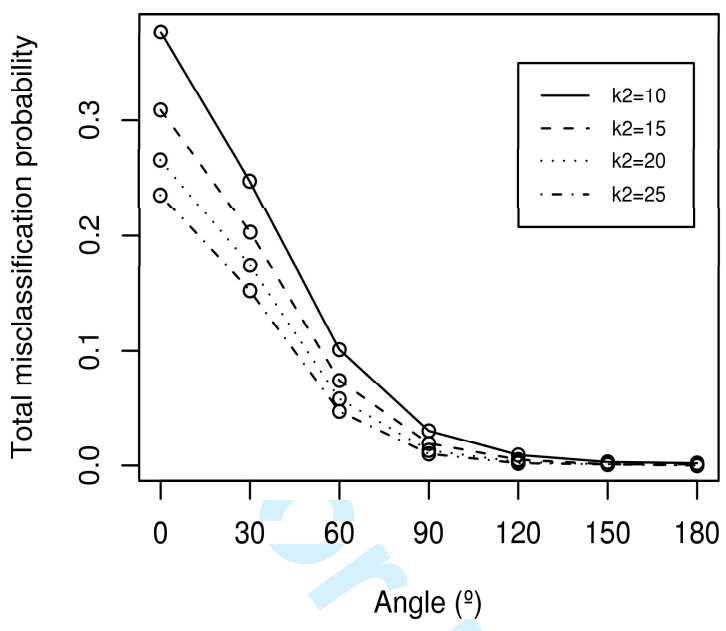

Dimension of the sphere 10

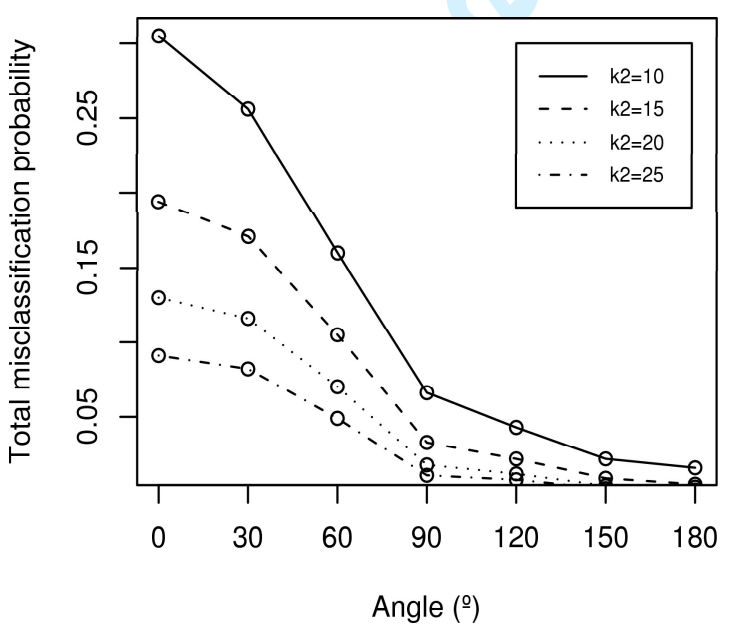

Dimension of the sphere 3

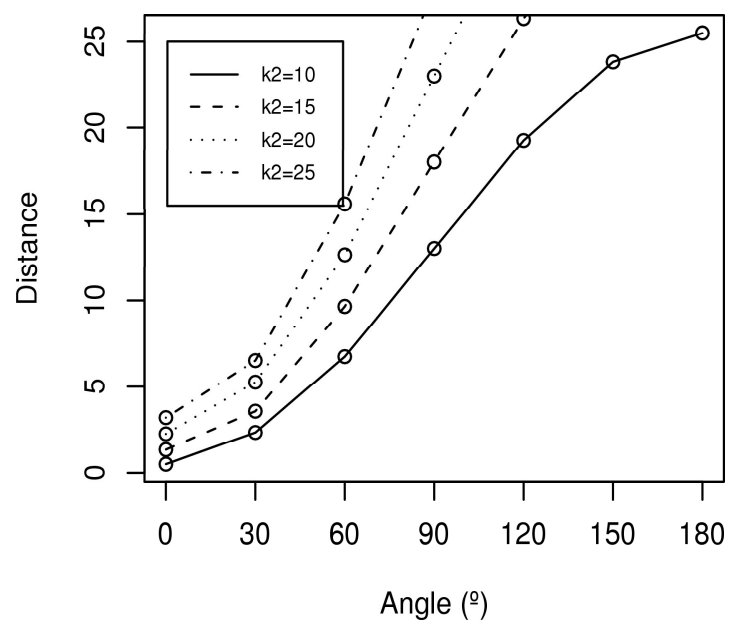

Dimension of the sphere 10

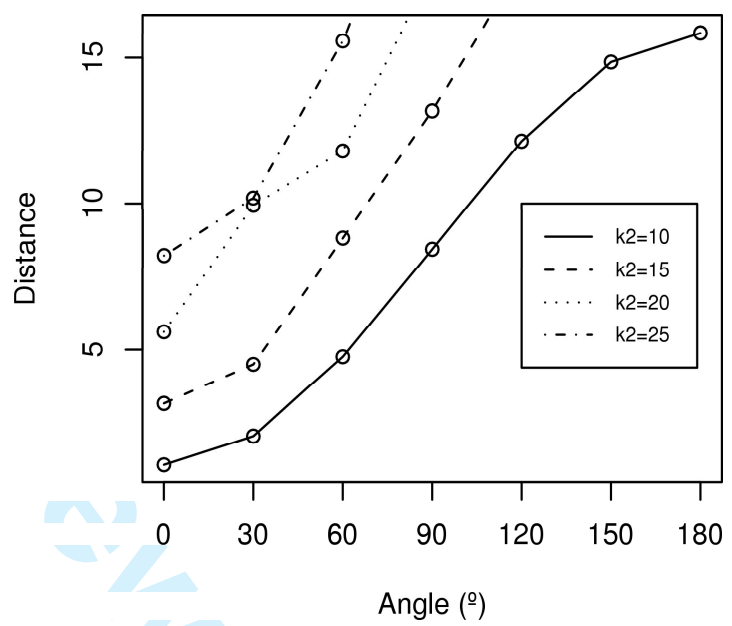

Figure 2: Estimate of the total misclassification probability and distance $D$ for $\kappa_{1}=5$. and also to estimate the parameters of the mixture of two Fisher distributions. This algorithm was already applied to these data in Figueiredo (2008). The partition of data obtained is the following: the group 1 contains the observations nos. 9,10,11,12,14,16,23,24,30 and the group 2 contain the remaining observations. We note that these two groups obtained 
with the EM algorithm are the same groups suggested in Wood (1982).

Before applying discriminant analysis to these data, we check informally through chi-square Q-Q plots whether the two groups come from the Fisher distribution, because if an observation comes from a Fisher distribution with large concentration parameter, then the approximation (2.4) holds. We use in this approximation the maximum likelihood estimates because the parameters of the populations are unknown. These estimates are given by $\widehat{\boldsymbol{\mu}}_{1}=(-0.318,0.548,0.736)^{\prime}, \widehat{\kappa}_{1}=21.93$ and $\widehat{\boldsymbol{\mu}}_{2}=(-0.623,0.104,0.775)^{\prime}, \widehat{\kappa}_{2}=33.97$ and were also obtained by the $E M$ algorithm. The chi-square Q-Q plots obtained for these data sets of size 9 and 24 are indicated in Fig.3. The fit to the Fisher distribution to each data set seems reasonable, and so for illustrating discriminant analysis, we have used these two samples.
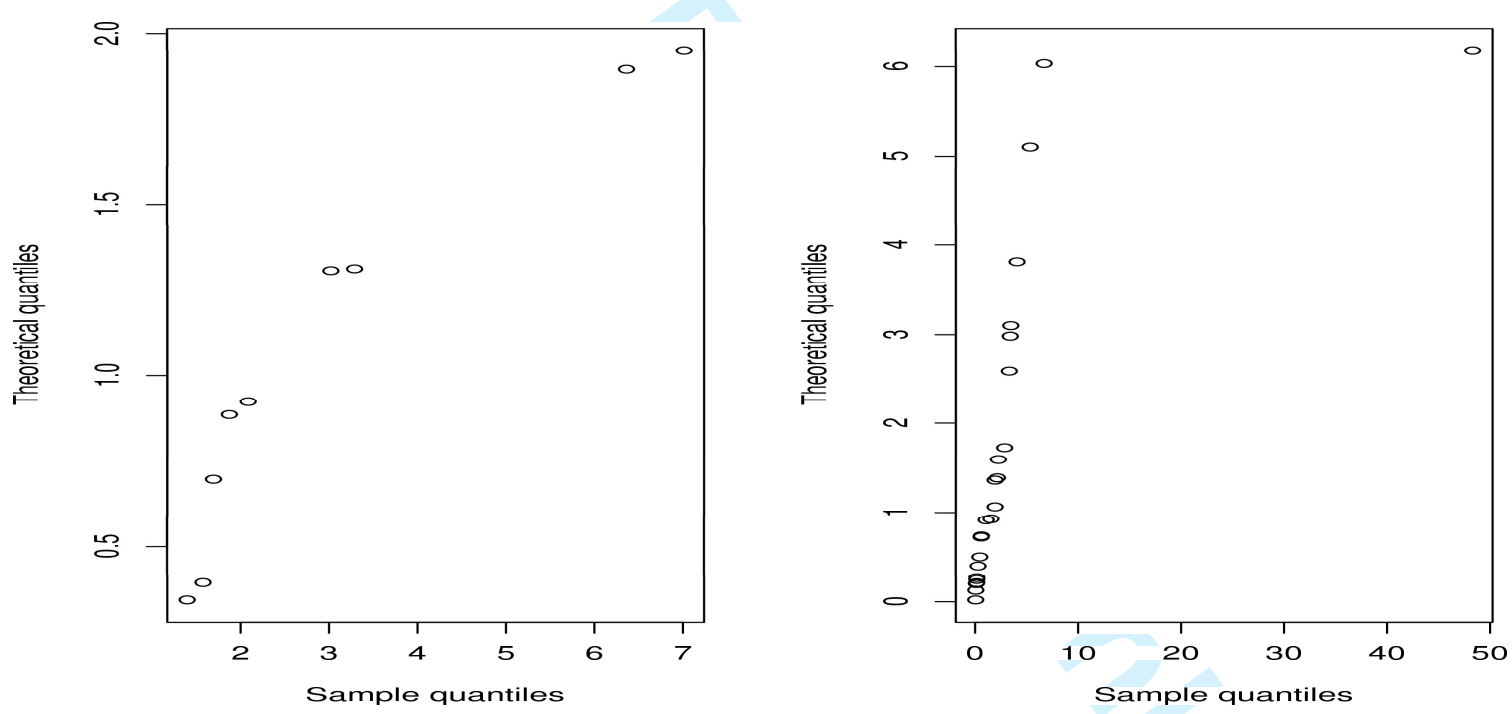

Figure 3: Chi-square Q-Q plots for the two samples

It is also important to check whether the two populations are distinct, before defining the discriminant rule. So, we have used the two-sample Watson-Williams test (Mardia and 
Jupp, 2000, p. 219) which enables us to test the equality of the mean directions of the two populations. The $F$-statistic test is equal to 21.579 and so, comparing with the $95 \%$ percentage point of $F_{2,62}$ distribution, which is equal to 3.145 , we reject the null hypothesis of equality of the two mean directions for the usual level of significance of $5 \%$. As this test assumes that the concentration parameters of the two populations are equal, we test this hypothesis using the test given in Mardia and Jupp, 2000, p. 220-221. The F-statistic test is equal to 0.8119 and comparing with the $97.5 \%$ percentage point of $F_{16,46}$ distribution, which is equal to 2.106 , we do not reject the null hypothesis of equality of the two concentration parameters for the usual level of significance of $5 \%$.

In the discriminant rule defined by (3.4), we suppose equal costs of misclassification and the parameters of the populations estimated by their maximum likelihood estimates. We obtain the following discriminant function:

$$
W_{12}=\left(\widehat{\kappa}_{1} \widehat{\boldsymbol{\mu}}_{1}-\widehat{\kappa}_{2} \widehat{\boldsymbol{\mu}}_{2}\right)^{\prime} \mathbf{x}+\ln \frac{c_{p}\left(\widehat{\kappa}_{2}\right) \widehat{\pi}_{2}}{c_{p}\left(\widehat{\kappa}_{1}\right) \widehat{\pi}_{1}}
$$

where we consider the following estimates for the prior probabilities: $\widehat{\pi}_{1}=0.284$ and $\widehat{\pi}_{2}=0.716$ also obtained through the $E M$ algorithm in Figueiredo (2008).

First, for validating the discriminant rule, we calculate the apparent error rate, obtained assigning the two samples using this discriminant rule. The apparent error rate is $1 / 33 \simeq 3 \%$; only the observation no. 1 was assigned wrongly. This error rate is biased, as the samples used for obtaining the rule are assigned with the rule. So, next we determine the cross-validation error rate based on the "Leave-One-Out" (LOO) method proposed by Lachenbruch and Mickey (1968). The LOO method consists in the following: we remove one observation from one of the samples and based on the remaining observations we obtain the rule, which is used for assigning the removed observation; next, we remove another observation of the sample, and we determine the rule, which is used for assigning the removed 
observation and we repeat the process until all observations of the two samples have been assigned. The error rate is the proportion of removed observations assigned incorrectly, and we obtained for this error rate $1 / 33 \simeq 3 \%$. Again, the observation which is not assigned correctly was the observation no. 1 . The distance $D$ given by (3.7) with the parameters replaced by their maximum likelihood estimates is equal to 8.361 . As the angle between the estimates of the mean directions is approximately $34^{\circ}$, we can say that the value 8.361 is not large, because based on the simulation study (Sec. 4.2), we know that the distance between the two populations is not very large for a small angle $\left(30^{\circ}\right)$ and this distance increases as the angle increases.

\section{References}

[1] Dempster, A. P.; Laird, N. M. and D. B. Rubin (1977). Maximum likelihood from incomplete data via the EM algorithm (with discussion), Journal of the Royal Statistical Society, B, 3, 1-38.

[2] El Khattabi, S. and F. Streit (1996). Identification analysis in directional statistics. Computational Statistics and Data Analysis 23, 45-63.

[3] Figueiredo, A. and P. Gomes (2006). Discriminant analysis based on the Watson distribution defined on the hypersphere. Statistics $\mathbf{4 0 , 5 , 4 3 5 - 4 4 5 .}$

[4] Figueiredo, A. (2008). Clustering estimates of a magnetic pole position of the earth. Submitted for publication.

[5] Fisher, N. I., Lewis, T. and B. J. J. Embleton (1987). Statistical analysis of spherical data, Cambridge University Press. 
[6] Fisher, N. I. (1993). Statistical analysis of circular data, Prentice Hall.

[7] Hill, G. W. (1977). Algorithm 518: Incomplete Bessel function I0(x); the von Mises distribution, ACM Trans. Math. Software, 3, 279-284.

[8] Jammalamadaka, S. R. and A. SenGupta (2001). Topics in Circular Statistics. World Scientific: Singapore.

[9] Johnson, R. A. and Wichern (1992). Applied Multivariate Statistical Analysis, 3rd ed, Cambridge University Press.

[10] Lachenbruch, P. A. and M. R. Mickey (1968). Estimation of error rates in Discriminant Analysis, Technometrics, 10, 1-11.

[11] Mardia, K. V. and P. E. Jupp (2000). Directional Statistics. John Wiley and Sons, Chichester.

[12] Morris, J. E. and P. J. Laycock (1974). Discriminant analysis of directional data. Biometrika 61, 2, 335-341.

[13] Schmidt, P. W. (1976). The non-uniqueness of the Australian Mesozoic palaeomagnetic pole position. Geophys. J. R. Astr. Soc., 47, 285-300.

[14] Stephens, M. A. (1982). Use of von Mises distribution to analyse continuous proportions. Biometrika, 69, 1, 197-203.

[15] Stephens, M. A. (1992). On Watson's ANOVA for directions: In K.V. Mardia (Ed.). The Art of Statistical Science, 75-85 (John Wiley and Sons Ltd).

[16] Watson, G. S. (1983). Statistics on spheres. John Wiley and Sons, New York.

[17] Wood, A. (1982). A bimodal distribution on the sphere, Applied Statistics, 31, 1, 52-58. 
[18] Wood, A. (1994). Simulation of the von Mises-Fisher distribution. Communications in Statistics - Simulation and Computation 23, 1, 157-164. 